\title{
Expression of Bcl-2 and Bad in hippocampus of status epileptic rats and molecular mechanism of intervened recombinant human erythropoietin
}

\author{
JIANGHUA YU ${ }^{1 *}$, ZHIQIN SHI $^{2 *}$, XUDONG SU $^{1}$, YI ZHOU $^{1}$, BIN LI $^{1}$, SHAN WANG $^{1}$, LIJING JIA ${ }^{1}$, \\ BO ZHAO ${ }^{2}$, MENGCHU ZHU ${ }^{2}$, XIAOHONG FENG $^{2}$, KUOCHANG YIN $^{1}$ and WEIPING WANG ${ }^{1}$ \\ ${ }^{1}$ Department of Neurology, The Second Hospital of Hebei Medical University; ${ }^{2}$ Department of \\ Experimental Diagnostics, Hebei Medical University, Shijiazhuang, Hebei 050000, P.R. China
}

Received March 30, 2018; Accepted May 18, 2018

DOI: $10.3892 / \mathrm{etm} .2018 .6250$

\begin{abstract}
Injury of hippocampal neurons in status epilepticus (SE) SD rats kindled by pentylenetetrazol (PTZ) were studied, and the changes of apoptosis neurons, protein expression of Bad and $\mathrm{Bcl}-2$ alone and combined application of phosphatidyl inositol 3-kinase (PI3K) inhibitor LY294002 and recombinant human erythropoietin (rHuEpo) were evaluated for the possible mechanisms of rHuEpo. The SE rats kindled by the PTZ were randomly divided into normal control group [normal saline (NS)], model group (PTZ + NS), rHuEpo treated group (PTZ + rHuEpo), LY294002 treated group (PTZ + LY294002 + rHuEpo) and LY294002 control group (rHuEpo + PTZ + DMSO). Apoptosis of hippocampal neurons was detected by TUNEL method; expression of phosphorylation protein kinase $\mathrm{B}$ (p-PKB/p-Akt), Bcl-2 and Bad were detected by immunohistochemistry; the expression of Bcl-2 mRNA, Bad mRNA in hippocampal neurons of rats were detected through reverse transcription polymerase chain reaction (RT-PCR); the expression of Akt, p-Akt and Bcl-2, Bad protein in hippocampal neurons of rats were detected by western blotting. The amount of apoptotic neurons was less in the rHuEpo treated group and the LY294002 control group than in the LY294002 treated group $(\mathrm{P}<0.05)$. The expression of p-Akt protein and Bcl-2 protein increased while the Bad protein decreased significantly in the rHuEpo treated group and the LY294002 control group compared with the LY294002 treated group $(\mathrm{P}<0.05)$. The expression of Bad protein and Bad mRNA in hippocampus increased while the p-Akt, Bcl-2,
\end{abstract}

Correspondence to: Dr Zhiqin Shi, Department of Experimental Diagnostics, Hebei Medical University, 361 Zhongshan East Road, Shijiazhuang, Hebei 050000, P.R. China

E-mail: hgn6m8@163.com

${ }^{*}$ Contributed equally

Key words: status epilepticus, recombinant human erythropoietin, phosphorylated protein kinase B, Bcl-2, Bad
Bcl-2 mRNA decreased significantly in the LY294002 treated group compared with the rHuEpo treated group $(\mathrm{P}<0.05)$. The $\mathrm{PI} 3 \mathrm{~K} / \mathrm{Akt}$ signaling pathway is one of the pathways of $\mathrm{rHuEpo}$ neuroprotective effects and was confirmed from both the of positive and negative aspects. rHuEpo regulates the expression of mitochondrial apoptotic pathway related factors Bad and Bcl-2 to inhibit apoptosis and promotes neuronal survival.

\section{Introduction}

Epilepsy is common among people of any age, region and race. There are various manifestations of epilepsy, and status epilepticus (SE) can occur in all kinds of epilepsy. Apoptosis is a major way of neuron loss after SE (1). Some studies have confirmed that epileptic seizures could cause damage of hippocampal neurons, and this damage might be one of the most important factors in the reconstruction of loop during recurrent seizures (2). The phosphatidyl inositol 3-kinase (PI3K)/Akt pathway may be an important signal transduction pathway for epileptic seizures. Bcl-2 and Bad belong to anti-apoptotic and pro-apoptotic proteins respectively, which play important roles in the mitochondrial apoptosis pathway. Recent studies have found that erythropoietin (Epo) is not merely a hematopoietic factor in a conventional way, but rather an important factor with multiple functions. The results of many animal models with nervous system damage have shown that Epo may reduce brain damage and improve nervous system function by reducing apoptosis and promoting survival (3-7). However, the anti-apoptotic mechanism of Epo in animal models of SE is unclear. In this study, we used pentylenetetrazol (PTZ) to induce epileptic seizures in rats to establish the SE model. The effects of Epo on neuronal apoptosis after epileptic seizures induced by PTZ, and on the expression of mitochondrial-apoptosis-pathway-related regulator Bcl-2 and Bad were observed, as well as whether the PI3K/Akt pathway was an important approach of neuroprotective effect of Epo to reduce apoptosis and promote survival.

\section{Materials and methods}

Experimental animals. A group of healthy adult rats (all male, 3-4 months) of Sprague-Dawley (SD) strain from Hebei 
Experimental Animal Center were selected. The range of initial weight was $220 \pm 20 \mathrm{~g}$. Attempts were made to minimize the number of animals used. Rats were normally fed with food and water and group-housed in plastic cages on a standard $12 / 12 \mathrm{~h} \mathrm{ligh/dark}$ cycle. The temperature was maintained within a normal range.

Main experimental reagents and equipments. Dimethyl sulphoxide (DMSO), LY294002 and PTZ (Sigma-Aldrich, St. Louis, MO, USA), recombinant human erythropoietin (rHuEpo) (North China Pharmaceutical Group Jintan Co., Ltd., Shijiazhuang, China), mouse monoclonal anti-rat phospho-Thr308-Akt, rat Bcl-2, Bad polyclonal antibodies (cat. nos. sc-377457, sc-56015, sc-8044; all from Santa Cruz Biotechnology, Inc., Dallas, TX, USA), in situ apoptosis detection kit (Roche Diagnostics, Indianapolis, IN, USA), 7314F electroencephalograph (Neurofax; Nihon Kohden Corporation, Tokyo, Japan), SR-6N stereotaxic apparatus (Narishige, Tokyo, Japan), microscopes (Olympus Corporation, Tokyo, Japan), pathology image analysis software (Beijing University of Aeronautics and Astronautics Image Center, Beijing, China), RT-PCR reverse transcription and amplification reagents (Promega Corporation, Madison, WI, USA), primers (Sangon Biotech Co., Ltd., Shanghai, China), PCR instrument (Eppendorf, Hamburg, Germany), gel scanning analysis system (Syngene Europe, Cambrige, UK).

SE model preparation and grouping. A total of 197 rats were used to prepare SE model based on the accepted approach and dosage: at first, PTZ $20 \mathrm{mg} / \mathrm{kg}$ was given by intraperitoneal injection. Then $10 \mathrm{mg} / \mathrm{kg}$ PTZ was injected each time at the interval of 10 min until generalized epileptic seizures and/or SE occurred. The performance of rats after administration was evaluated according to Racine' scale. Grade 0 , no abnormal performance; grade I, facial muscle twitch and/ or rhythmic mastication; grade II, regular nodding or wet dog shakes (WDS); grade III, clonus of a single forelimb; grade IV, clonus of both forelimbs with standing posture; grade $\mathrm{V}$, sudden fall and/or generalized tonic-clonic convulsion. When grade IV or V appeared, it was considered as a generalized seizure, and regarded as SE when the duration of onset was $30 \mathrm{~min}$ or more. In order to ensure experiment scale, rats of midway-death or unsuccessful models were removed and randomly supplemented. The final 125 successful models were randomly divided into 5 groups: normal control group [normal saline (NS)], model group (PTZ + NS), rHuEpo intervention group (PTZ + rHuEpo), LY294002 intervention group (PTZ + rHuEpo + LY294002) and LY294002 control group $(\mathrm{PTZ}+\mathrm{rHuEpo}+\mathrm{DMSO})$, with 25 rats in each group.

Drug administration. Model group: An intraperitoneal injection (i.p.) of $2 \mathrm{ml}$ of $0.9 \%$ sodium chloride solution at $30 \mathrm{~min}$ after SE was induced with PTZ.rHuEpo intervention group: an intraperitoneal injection of rHuEpo 5,000 U/ $\mathrm{kg}$ at $30 \mathrm{~min}$ after SE was induced with PTZ. LY294002 intervention group (with DMSO as solvent, LY294002 concentration at $10 \mu \mathrm{g} / 5 \mu \mathrm{l}$ ): an intraventricular injection of $5 \mu \mathrm{l}$ of LY294002 at $10 \mathrm{~min}$ after SE was induced with PTZ, followed by an intraperitoneal injection of rHuEpo 5,000 U/kg at 30 min after SE. LY294002 control group: an intraventricular injection of $5 \mu \mathrm{l}$ of DMSO at 10 min after SE was induced with PTZ, followed by an intraperitoneal injection of $\mathrm{rHuEpo} 5,000 \mathrm{U} / \mathrm{kg}$ at $30 \mathrm{~min}$ after SE. Normal control group: an intraperitoneal injection of $0.9 \%$ sodium chloride solution of the same volume.

Stereotaxic localization of lateral ventricle and administration in rats. The Rat Brain in Stereotaxic Coordinates was used as the standard to locate the injection site $(0.8 \mathrm{~mm}$ backward the anterior fontanelle, $1.5 \mathrm{~mm}$ aside the sagittal slit and $3.8 \mathrm{~mm}$ under the dura mater). First, SE was induced with PTZ. After 10 min, the LY294002 intervention group was injected with $5 \mu$ l of LY294002, while the LY294002 control group was injected with $5 \mu \mathrm{l}$ of DMSO. The needles were not drawn until 5 min after injection, while the behavior of the rat was observed.

Behavioral observation and electroencephalogram (EEG) of rats. Continuous behavioral observation lasted for $2 \mathrm{~h}$ after the drug administration. Whether there was seizure onset, manifestation and duration were recorded in detail.

EEG: Five rats in each group were randomly selected for EEG recording. An intraperitoneal injection of $4 \%$ chloral hydrate $(35 \mathrm{mg} / \mathrm{kg})$ was given for anaesthesia, and the rat skull was carefully exposed after fixed to the cephalostat. Eight leads were connected by 4 electrodes on the left and right hippocampus and cortex. The position of cortical electrode: $2 \mathrm{~mm}$ forward the anterior fontanelle, $2 \mathrm{~mm}$ aside to the midline and $2 \mathrm{~mm}$ in depth. The position of hippocampal electrode: $3.8 \mathrm{~mm}$ backward the anterior fontanelle, $2.4 \mathrm{~mm}$ aside to the middle line and $3.8 \mathrm{~mm}$ in depth. The electrodes were placed and fixed after drilling, with the reference electrode placed at the tip of the rat's nose. EEG machine parameters were set as follows: paper speed of $30 \mathrm{~mm} / \mathrm{sec}$, sensitivity of $20 \mu \mathrm{V} / \mathrm{mm}$, wave filtering of $15 \mathrm{~Hz}$, time constant of 0.1 . EEG was recorded after the rat awoke from anesthesia.

\section{Observation of hippocampal histology}

Specimen preparation. At $24 \mathrm{~h}$ after onset of SE, excessive anesthesia was applied. After exposure of the heart, the aorta was intubated from the left ventricle, and the opening of the right atrial appendage made the perfusion fluid flow out smoothly. After connected to the perfusion system, NS and $4 \%$ paraformaldehyde were injected successively, each in the volume of $500 \mathrm{ml}$. Then the brain tissue was quickly taken out after craniotomy and vertically transected at the optic chiasma and mammillary bodies. The middle-segment brain tissue containing hippocampus and intraventricular injection site was fully soaked in the solution of sufficient polyformaldehyde for $24 \mathrm{~h}$ at $4^{\circ} \mathrm{C}$ followed by dehydration and embedding. The tissue was continuously cut into slices all the way to the hippocampal level at the thickness of $5 \mu \mathrm{m}$ from coronal position. The sections were prepared for TUNEL and immunohistochemical staining.

Histopathological observation of hippocampus. i) TUNEL staining by TUNEL kit. After the sections were deparaffinized and rehydrated, the proteinase $\mathrm{K}$ (which does not contain deoxyribonuclease) was added for incubation. After full washing with PBS, the conversion agent POD was added, followed by the steps of color rendering, redyeing and covering. All the specimens were processed into 3 sections 
according to the methods above. In each of the hippocampal sections, 10 non-overlapping fields were selected by random, and the number of cells with dyed and visible nuclei was counted. The average value of 30 counts of each sample was taken as final result, and the average counts of samples in each group were obtained.

ii) Immunohistochemical staining: the Streptavidin-Perosidase method was employed. After deparaffinized and hydrated, the sections were washed with PBS 3 times, and 3\% $\mathrm{H}_{2} \mathrm{O}_{2}$ was used for quenching endogenous peroxidase activity. Normal low-lethal serum was used as a blocking buffer after PBS rinsing again. Mouse monoclonal anti-phospho-Thr308-Akt antibody (diluted 1:200), anti-Bcl-2 antibody (diluted 1:200), anti-Bad antibody (diluted 1:150) were added and placed at $4^{\circ} \mathrm{C}$ overnight, and biotinylated goat anti-rabbit IgG was added at room temperature after PBS flushing. Incubation, color rendering, redyeing and covering were sequentially carried out. PBS was used to replace Bcl-2 and Bad polyclonal antibodies in the control group. The observation target was the expression of Bcl-2 and Bad in hippocampal tissues. All the specimens were processed into 3 sections according to the method above. In each of the hippocampal sections, 10 non-overlapping fields were selected randomly, and the number of cells with dyed and visible nuclei was counted. The average value of 30 counts of each sample was taken as final result, and the average count of samples in each group was obtained.

RT-PCR detection. Ten rats were randomly selected from each group and the hippocampus was quickly taken out after death. The total RNA of each rat's hippocampus was extracted according to the instruction of TRIzol reagent, and the purity and concentration were measured by ELISA microplate reader. A total of $2 \mu \mathrm{g}$ of each sample was used for reverse transcription into its complementary DNA, and $1 \mu \mathrm{g}$ of the complementary DNA was used as substrate for PCR amplification. The primers were customized at Sangon Biotech Co., Ltd. A total of $5 \mu \mathrm{l}$ of each reverse transcriptase PCR (RT-PCR) amplification product was observed after electrophoresis on $2 \%$ agarose gel, and the density of each band was analyzed on the gel image analysis system to obtain semi-quantitative results. The statistical analysis was carried out after 5 repeated average calculations. The relative content of the product was exhibited by the ratio of $\mathrm{Bad} / \beta$-actin and $\mathrm{Bcl}-2 / \beta$-actin.

Western blotting. In each group, 10 experimental rats were randomly selected. After rapid sacrifice, the hippocampal tissues were rapidly removed. The total protein was extracted and the concentration was determined (BCA method). After the protein were denatured, electrically transferred and sealed, mouse monoclonal anti-phospho-Thr308-Akt antibody (1:300), anti-Bcl-2 antibody (1:200) and anti-Bad antibody (1:150) were added into $50 \mu \mathrm{g}$ of protein and placed at $4^{\circ} \mathrm{C}$ overnight. After TPBS bleaching, the rabbit anti-mouse Igg monoclonal antibody (1:3,000; cat. no. 58802; Cell Signaling Technology, Danvers, MA, USA) was added. After incubation for $1 \mathrm{~h}$, the target was rinsed with TPBS and PBS, respectively. The target strip and the $\beta$-actin strip were scanned and the unit density was measured. The ratio of the two was calculated and analyzed statistically, then repeated 5 times to calculate the mean value and carry out final statistical analysis.
Statistical analysis. All the data collected in the experiment are expressed as the mean \pm standard deviation. The data were analyzed by SPSS 21 software (IBM Corp., Armonk, NY, USA). One-way ANOVA with Student-Newman-Keuls post hoc test was used for multiple comparisons among the groups. $\mathrm{P}<0.05$ was considered to indicate a statistically significant difference.

\section{Results}

Behavioral changes in rats. A total of 197 rats were injected with PTZ. The minimum dose and quantity distribution of convulsion were $30 \mathrm{mg} / \mathrm{kg}$ (75), $40 \mathrm{mg} / \mathrm{kg}$ (77), and $50 \mathrm{mg} / \mathrm{kg}(45)$. The main manifestations of convulsion are rhythmic head retraction and myoclonus. The injection dose of PTZ increased rapidly with the time gradient, rapidly triggering the twitching of the limbs of the rats. The self-regulation ability of the body posture was quickly lost in the stage of generalized tonic-clonic seizures. Severe contraction of the extensors in the anterior and hind limbs of the rats were observed and respiratory depression appeared. Hyperemia of eyeball and blue around the lips were also observed. Within a few minutes, generalized tonic-clonic convulsion occurred with the SE duration of $30 \mathrm{~min}$ or more. Approximately $35 \%$ rats died during the process, and 3 unsuccessful modeling rats were removed and randomly supplemented. No behavioral changes were found in the normal control group.

Changes of EEG. The EEG of the model group showed a large number of paroxysmal high amplitude spike waves, sharp waves, sharp slow/spike slow complex waves. The frequency and/or amplitude of epileptic seizures in the rHuEpo intervention the LY294002 intervention and the LY294002 control groups were significantly lower than those in the model group, which indicated the epileptic discharge was significantly inhibited. Compared with the LY294002 intervention group, epileptic discharges in the rHuEpo intervention group and the LY294002 control group decreased significantly.

TUNEL staining results (Table I). Apoptotic cells are TUNEL-positive cells featured with brown yellow nuclei under the light microscope. There was significant difference for TUNEL-positive cells $(\mathrm{F} 4,45=19.19, \mathrm{P}<0.05)$ among the five groups. In the normal control group, there were few apoptotic cells in brain tissue slices, while in the other groups, apoptotic cells were distributed in hippocampal areas to a different degree. The number of apoptotic cells in the normal control group was significantly less than that in the other 4 groups $(\mathrm{P}<0.05)$. The frequency of apoptotic cells in the slices of the model group was significantly increased, with the nuclei fixed in a circular or irregular shape. Under high power microscope, chromatin staining, aggregation, fragmentation and other phenomena can be observed, which is consistent with the morphological characteristics of apoptotic cells. The number of apoptotic cells in the rHuEpo intervention, the LY294002 intervention and the LY294002 control groups was significantly less than that in the model group $(\mathrm{P}<0.05)$. The number of apoptotic cells in the rHuEpo intervention group and the LY294002 control group was significantly less than that in the 
Table I. The count of apoptotic cells, p-Akt-positive cells and $\mathrm{p}$-Akt protein of hippocampus area in each group (mean \pm standard deviation, $\mathrm{n}=10$ ).

\begin{tabular}{lccc}
\hline Groups & $\begin{array}{c}\text { Apoptotic } \\
\text { cells }\end{array}$ & $\begin{array}{c}\text { p-Akt-positive } \\
\text { cells }\end{array}$ & p-Akt \\
\hline Control group & $7.23 \pm 1.05$ & $21.41 \pm 1.20$ & $0.398 \pm 0.064$ \\
Model group & $26.72 \pm 3.53^{\mathrm{a}}$ & $26.57 \pm 2.46^{\mathrm{a}}$ & $0.528 \pm 0.044^{\mathrm{a}}$ \\
rHuEpo & $16.59 \pm 1.12^{\mathrm{a}-\mathrm{c}}$ & $54.81 \pm 5.08^{\mathrm{a}-\mathrm{c}}$ & $0.759 \pm 0.027^{\mathrm{a}-\mathrm{c}}$ \\
treated group & & & \\
LY294002 & $21.08 \pm 2.67^{\mathrm{a}, \mathrm{b}}$ & $33.07 \pm 4.87^{\mathrm{a}, \mathrm{b}}$ & $0.64 \pm 0.012^{\mathrm{a}, \mathrm{b}}$ \\
treated group & & & \\
LY294002 & $17.47 \pm 1.86^{\mathrm{a}-\mathrm{c}}$ & $52.35 \pm 4.63^{\mathrm{a}-\mathrm{c}}$ & $0.756 \pm 0.027^{\mathrm{a}-\mathrm{c}}$ \\
control group & & & \\
F-value & 19.19 & 28.77 & 15.70 \\
P-value & $<0.05$ & $<0.05$ & $<0.01$ \\
\hline
\end{tabular}

${ }^{a} \mathrm{P}<0.05$, control group vs. model, rHuEpo treated, LY294002 treated, LY294002 control groups; ${ }^{b} \mathrm{P}<0.05$, model group vs. rHuEpo treated, LY294002 treated, LY294002 control groups; ${ }^{\text {P }}<0.05$, LY294002 treated group vs. rHuEpo treated, LY294002 control groups. No significant difference was found between rHuEpo treated group and LY294002 control group. rHuEpo, recombinant human erythropoietin.

Table II. The count of Bcl-2 positive cells, Bcl-2 mRNA, Bcl-2 protein of rats hippocampus area in each group (mean \pm standard deviation, $\mathrm{n}=10$ ).

\begin{tabular}{lccc}
\hline & $\begin{array}{c}\text { Bcl-2 positive } \\
\text { cells }\end{array}$ & Bcl-2 mRNA & Bcl-2 \\
\hline Groups & $\begin{array}{l}\text { Control group } \\
23.197 \pm 2.726\end{array}$ & $0.440 \pm 0.076$ & $0.349 \pm 0.049$ \\
$\begin{array}{l}\text { Model group } \\
\text { rHuEpo }\end{array}$ & $29.606 \pm 2.143^{\mathrm{a}}$ & $0.605 \pm 0.051^{\mathrm{a}}$ & $0.508 \pm 0.044^{\mathrm{a}}$ \\
treated group & $42.639 \pm 1.829^{\mathrm{a}-\mathrm{c}}$ & $0.984 \pm 0.019^{\mathrm{a}-\mathrm{c}}$ & $0.791 \pm 0.045^{\mathrm{a}-\mathrm{c}}$ \\
$\begin{array}{l}\text { LY294002 } \\
\text { treated group }\end{array}$ & $35.415 \pm 2.011^{\mathrm{a}, \mathrm{b}}$ & $0.843 \pm 0.034^{\mathrm{a}, \mathrm{b}}$ & $0.636 \pm 0.021^{\mathrm{a}, \mathrm{b}}$ \\
$\begin{array}{l}\text { LY294002 } \\
\text { control group }\end{array}$ & $41.060 \pm 0.476^{\mathrm{a}-\mathrm{c}}$ & $0.981 \pm 0.021^{\mathrm{a}-\mathrm{c}}$ & $0.762 \pm 0.050^{\mathrm{a}-\mathrm{c}}$ \\
F-value & 19.27 & 27.95 & 18.07 \\
P-value & $<0.05$ & $<0.05$ & $<0.05$ \\
\hline
\end{tabular}

${ }^{\text {aP }}<0.05$, control group vs. model group, rHuEpo treated, LY294002 treated, LY294002 control groups; ${ }^{b} \mathrm{P}<0.05$, model group vs. rHuEpo treated, LY294002 treated, LY294002 control groups; ${ }^{\text {P }}<0.05$, LY294002 treated group vs. rHuEpo treated, LY294002 control groups. No significant difference was found between rHuEpo treated group and LY294002 control group. rHuEpo, recombinant human erythropoietin.

LY294002 intervention group $(\mathrm{P}<0.05)$. The number of apoptotic cells in the LY294002 control group was more than that in the rHuEpo intervention group, but there was no statistical difference $(\mathrm{P}>0.05)$.

Immunohistochemical staining results. The expression of $\mathrm{p}-\mathrm{Akt}$, Bcl-2 and Bad in hippocampus (Tables I-III; Figs. 1 and 2).

Brown yellow is positive for staining, mainly located in cytoplasm, with a small amount in nuclei and membranes. There was significant difference for the number of p-Akt-IR
Table III. The count of Bad positive cells, Bad mRNA, Bad protein of rats hippocampus area in each group (mean \pm standard deviation, $\mathrm{n}=10$ ).

\begin{tabular}{|c|c|c|c|}
\hline Groups & $\begin{array}{l}\text { Bad-positive } \\
\text { cells }\end{array}$ & Bad mRNA & Bad \\
\hline Control group & $16.931 \pm 1.158$ & $0.350 \pm 0.041$ & $0.291 \pm 0.033$ \\
\hline Model group & $43.804 \pm 4.386^{\mathrm{a}}$ & $0.728 \pm 0.076^{\mathrm{a}}$ & $0.668 \pm 0.045^{\mathrm{a}}$ \\
\hline $\begin{array}{l}\text { rHuEpo } \\
\text { treated group }\end{array}$ & $24.060 \pm 0.807^{\mathrm{a}-\mathrm{c}}$ & $0.493 \pm 0.013^{\mathrm{a}-\mathrm{c}}$ & $0.408 \pm 0.017^{\mathrm{a}-\mathrm{c}}$ \\
\hline $\begin{array}{l}\text { LY294002 } \\
\text { treated group }\end{array}$ & $35.424 \pm 2.290^{\mathrm{a}, \mathrm{b}}$ & $0.608 \pm 0.014^{\mathrm{a}, \mathrm{b}}$ & $0.542 \pm 0.021^{\mathrm{a}, \mathrm{b}}$ \\
\hline $\begin{array}{l}\text { LY294002 } \\
\text { control group }\end{array}$ & $24.308 \pm 0.677^{\mathrm{a}-\mathrm{c}}$ & $0.495 \pm 0.013^{\mathrm{a}-\mathrm{c}}$ & $0.454 \pm 0.026^{\mathrm{a}-\mathrm{c}}$ \\
\hline F-value & 21.00 & 13.12 & 22.07 \\
\hline P-value & $<0.05$ & $<0.05$ & $<0.05$ \\
\hline
\end{tabular}

${ }^{\text {aP }}<0.05$, control group vs. model, rHuEpo treated, LY294002 treated, LY294002 control groups; ${ }^{b} \mathrm{P}<0.05$, model group vs. rHuEpo treated, LY294002 treated, LY294002 control groups; ${ }^{\mathrm{P}}<0.05$, LY294002 treated group vs. rHuEpo treated, LY294002 control groups. No significant difference was found between rHuEpo treated group and LY294002 control group. rHuEpo, recombinant human erythropoietin.

cells $(\mathrm{F} 4,45=28.77, \mathrm{P}<0.05), \mathrm{Bcl}-2-\mathrm{IR}$ cells $(\mathrm{F} 4,45=19.27$, $\mathrm{P}<0.05)$ and Bad-IR cells $(\mathrm{F} 4,45=21.00, \mathrm{P}<0.05)$ among the five groups. In the cytoplasm of the normal control group, there were occasionally scattered brown yellow particles in the cytoplasm. Compared with the normal control group, the number of p-Akt-, Bcl-2- and Bad-positive cells in the other 4 groups showed a significant increase, showing statistical difference $(\mathrm{P}<0.05)$. The number of p-Akt- and Bcl-2-positive cells in the rHuEpo intervention group, the LY294002 intervention group and the LY294002 control group was significantly more than that in the model group, while the number of Bad-positive cells was significantly less than that in the model group, showing statistical difference $(\mathrm{P}<0.05)$. Compared with the LY294002 intervention group, the number of p-Akt- and Bcl-2-positive cells in the rHuEpo intervention group and the LY294002 control group was more, but the number of Bad-positive cells was less, showing statistical difference $(\mathrm{P}<0.05)$. Compared with the LY294002 control group, the number of p-Akt and Bcl-2-positive cells in the rHuEpo intervention group was more, but the number of Bad-positive cells was less, showing no statistical difference $(\mathrm{P}>0.05)$.

RT-PCR to determine the expression of Bcl-2 $m R N A$ and Bad $m R N A$ (Tables II and III; Figs. 3-5). There was significant difference for Bcl-2 mRNA (F4, 45=27.95, P<0.05) and Bad mRNA $(\mathrm{F} 4,45=13.12, \mathrm{P}<0.05)$ among the five groups. There were weak expressions of Bcl-2 mRNA and Bad mRNA in the control group. Compared with the control group, the expression of Bcl-2 mRNA and Bad mRNA in the model, the rHuEpo intervention, the LY294002 intervention and the LY294002 control groups all increased significantly $(\mathrm{P}<0.05)$. The expression of Bad mRNA in the model group was significantly higher than that in the rHuEpo intervention, the LY294002 intervention and the LY294002 control groups, and the expression of Bcl-2 mRNA was significantly decreased 

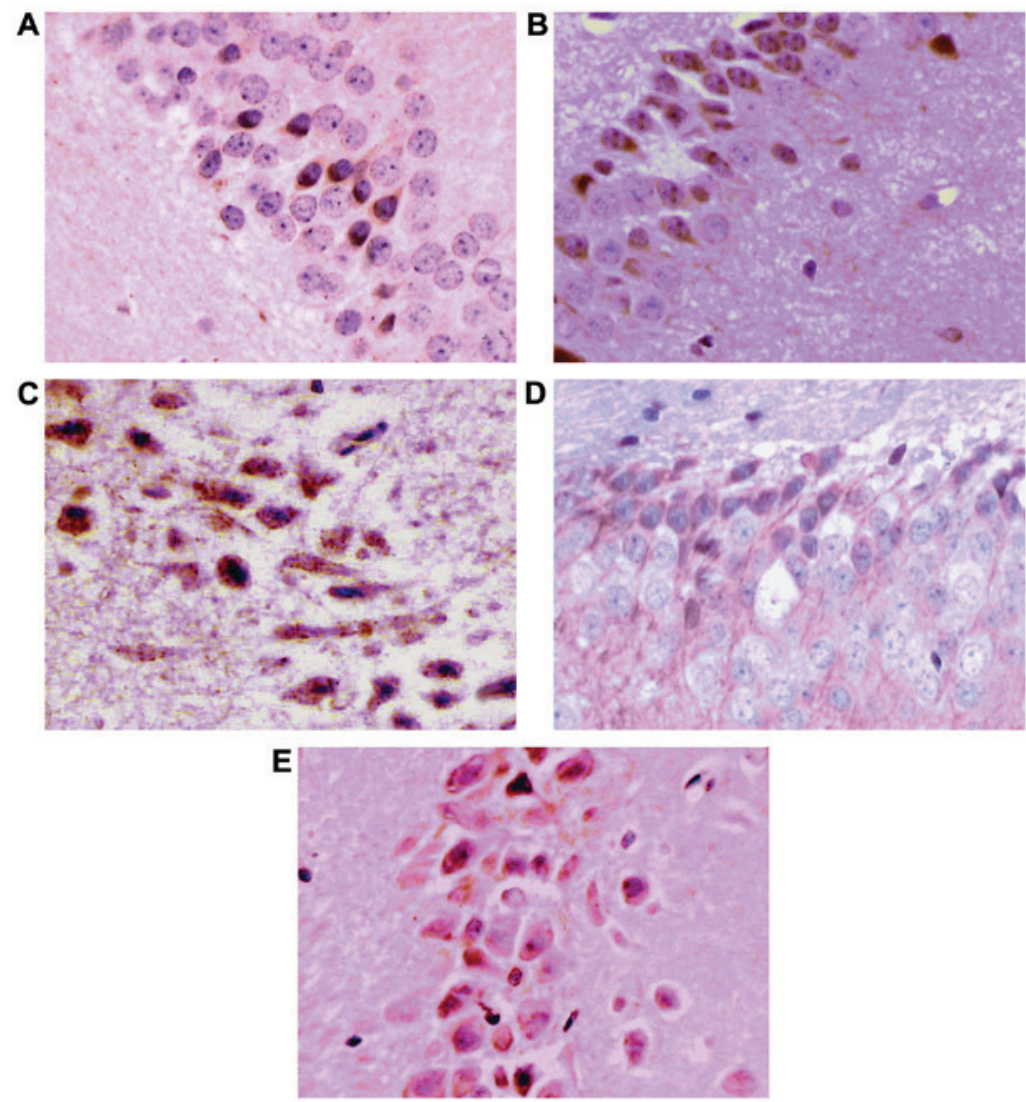

Figure 1. The expression of Bcl-2-positive cells in rat hippocampus (SP, x400). (A) Control group; (B) model group; (C) rHuEpo treated group; (D) LY294002 treated group; (E) LY294002 control group.
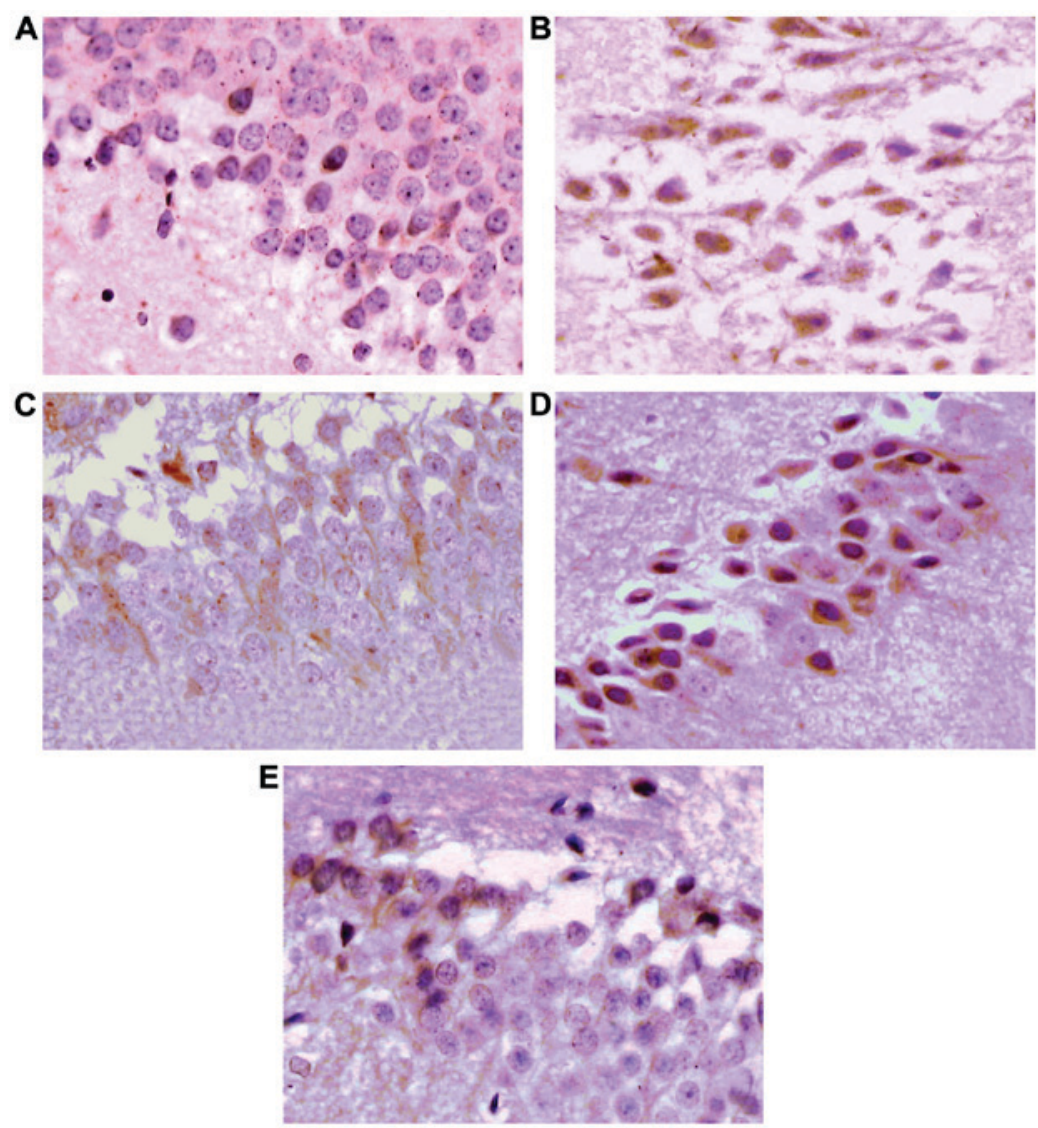

Figure 2. The expression of Bad-positive cells in rat hippocampus (SP, x400). (A) Control group; (B) model group; (C) rHuEpo treated group; (D) LY294002 treated group; (E) LY294002 control group. 


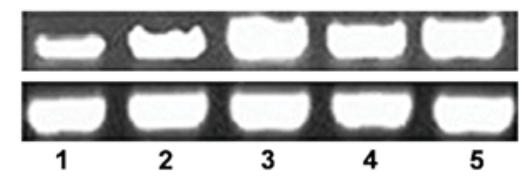

$\mathrm{Bcl}-2(446 \mathrm{bp})$

$\beta$-actin (375bp)
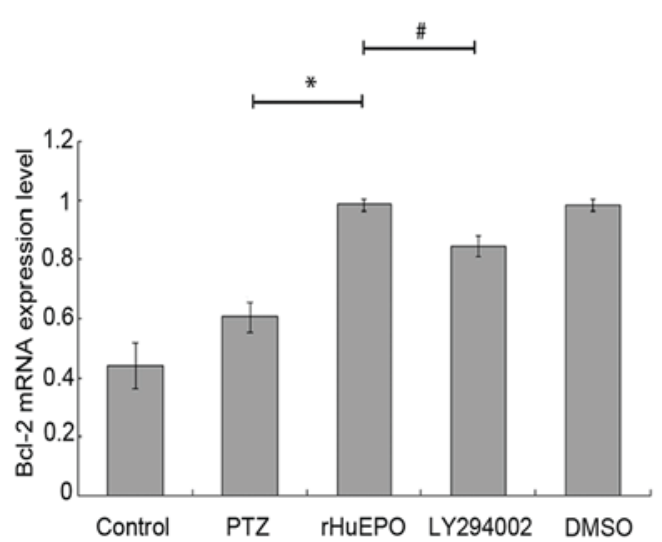

Figure 3. The expression of Bcl-2 mRNA in rat hippocampus of each group. Lane 1, control group; lane 2, model group; lane 3, rHuEpo treated group; lane 4, LY294002 treated group; lane 5, LY294002 control group. Model group vs. rHuEpo treated group ("p $<0.05)$; rHuEpo treated group vs. LY294002 treated group ( $\left.{ }^{\mathrm{p}}<<0.05\right)$. PTZ, pentylenetetrazol; rHuEpo, recombinant human erythropoietin; DMSO, dimethyl sulphoxide.

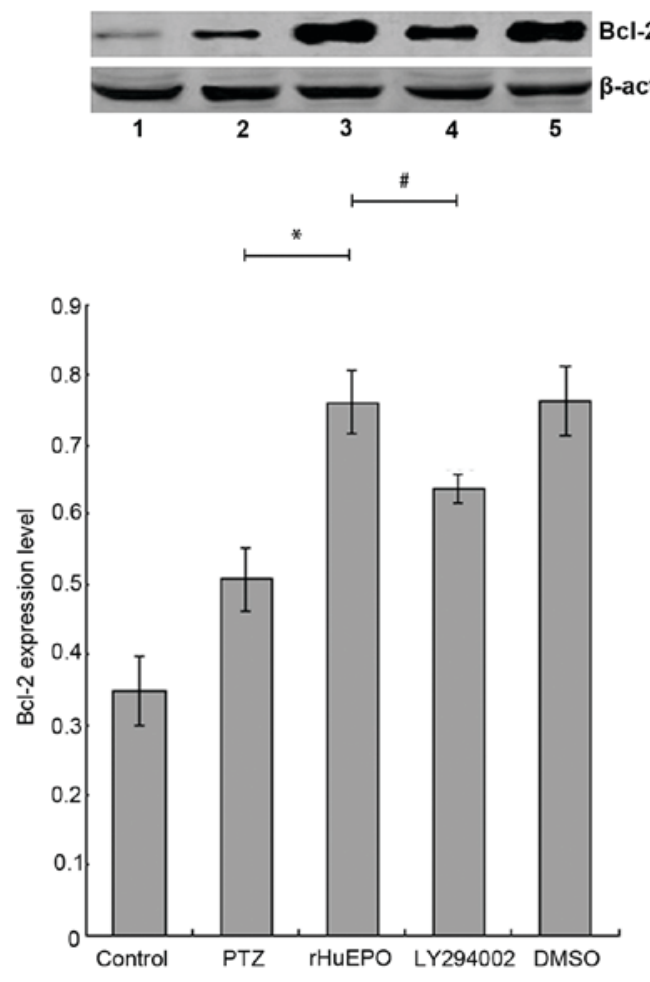

Figure 4. The expression of Bcl-2 protein in rat hippocampus of each group. Lane 1, control group; lane 2, model group; lane 3, rHuEpo treated group; lane 4, LY294002 treated group; lane 5, LY294002 control group. Model group vs. rHuEpo treated group ( $\left.{ }^{*} \mathrm{p}<0.05\right)$; rHuEpo treated group vs. LY294002 treated group ( $\left.{ }^{*} \mathrm{p}<0.05\right)$. PTZ, pentylenetetrazol; rHuEpo, recombinant human erythropoietin; DMSO, dimethyl sulphoxide.

$(\mathrm{P}<0.05)$. Compared with the LY294002 intervention group, the expression of Bad mRNA in the rHuEpo intervention group and LY294002 control group decreased significantly, and the
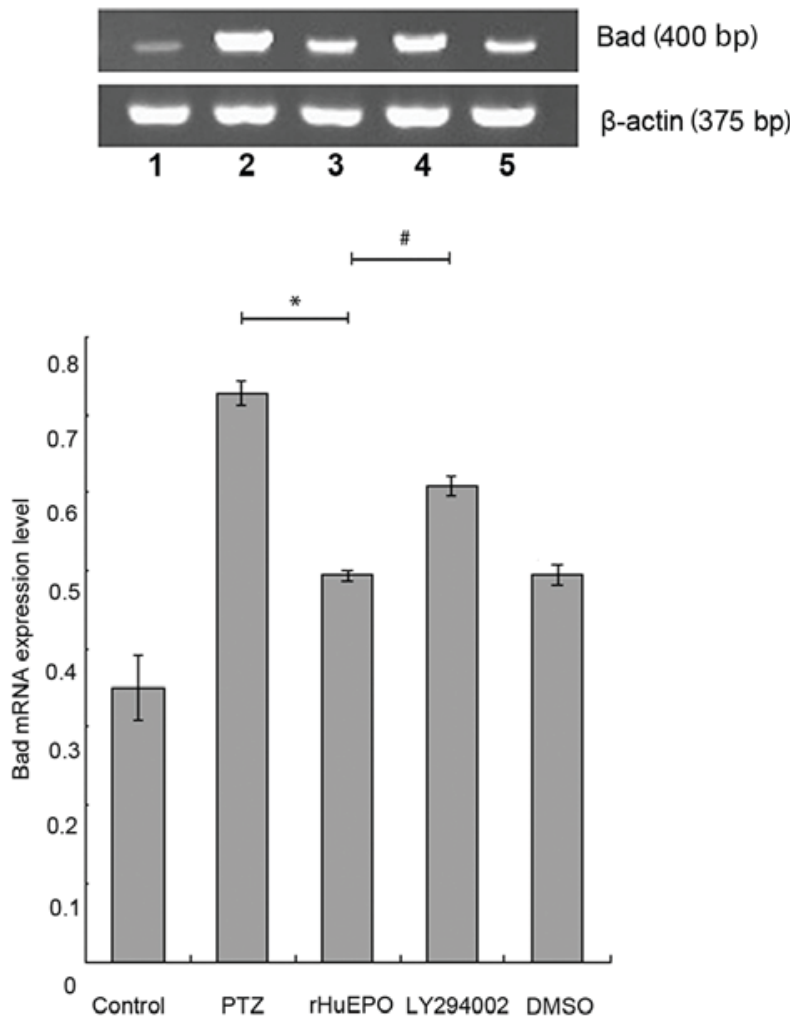

Figure 5. The expression of Bad mRNA in rat hippocampusof each group. Lane 1, control group; lane 2, model group; lane 3, rHuEpo treated group; lane 4, LY294002 treated group; lane 5, LY294002 control group. Model group vs. rHuEpo treated group $\left({ }^{*} \mathrm{p}<0.05\right)$; rHuEpo treated group vs. LY294002 treated group $\left({ }^{\sharp} \mathrm{p}<0.05\right)$. PTZ, pentylenetetrazol; rHuEpo, recombinant human erythropoietin; DMSO, dimethyl sulphoxide.

expression of Bcl-2 mRNA increased significantly $(\mathrm{P}<0.05)$. Compared with the LY294002 control group, the expression of Bad mRNA in the rHuEpo intervention group decreased and the expression of Bcl-2 mRNA increased, showing no statistical difference $(\mathrm{P}>0.05)$.

Western blotting to determine the expression of Akt, p-Akt, $B c l-2$ and Bad (Tables I-III; Figs. 4-6). The expression of Akt and p-Akt, Bcl-2 and Bad protein in the hippocampus of rats was detected by western blotting. There was significant difference for $\mathrm{p}-\mathrm{Akt}(\mathrm{F} 4,45=15.70, \mathrm{P}<0.01)$, Bcl-2 (F4, $45=18.07, \mathrm{P}<0.05)$ and $\mathrm{Bad}(\mathrm{F} 4,45=22.07, \mathrm{P}<0.05)$ among the five groups, while no significant difference was detected for total AKT between each experimental groups ( F4, 45=0.02, $\mathrm{P}>0.05)$. The results showed that compared with the model group, rHuEpo intervention group, LY294002 intervention group and LY294002 control group, the expression of p-Akt, $\mathrm{Bcl}-2$ and $\mathrm{Bad}$ in the control group increased significantly $(\mathrm{P}<0.05)$. Compared with the rHuEpo intervention group, the LY294002 intervention group and the LY294002 control group, the expression of p-Akt and Bcl-2 in the PTZ group was obviously decreased, and the expression of Bad was significantly increased $(\mathrm{P}<0.05)$. Compared with the LY294002 intervention group, the expression of $\mathrm{p}-\mathrm{Akt}$ and $\mathrm{Bcl}-2$ in the rHuEpo intervention group and the LY294002 control group increased significantly, and the expression of Bad protein decreased significantly, showing statistical difference $(\mathrm{P}<0.05)$. Although 

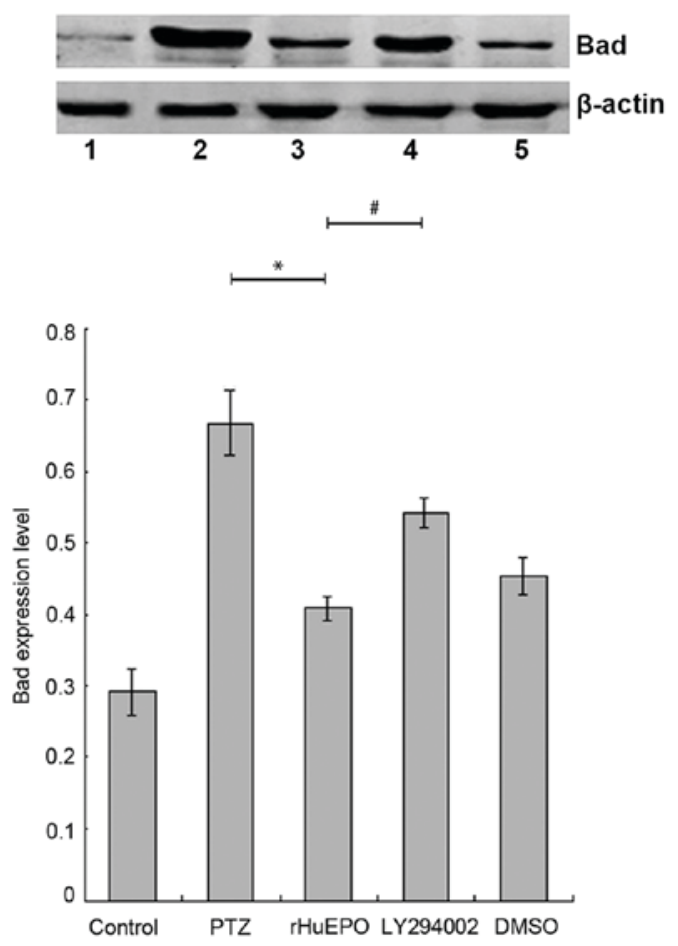

Figure 6. The expression of Bad protein in rat hippocampus of each group. Lane 1, control group; lane 2, model group; lane 3, rHuEpo treated group; lane 4, LY294002 treated group; lane 5, LY294002 control group. Model group vs. rHuEpo treated group ("p<0.05); rHuEpo treated group vs. LY294002 treated group $\left({ }^{\#} \mathrm{p}<0.05\right)$. PTZ, pentylenetetrazol; rHuEpo, recombinant human erythropoietin; DMSO, dimethyl sulphoxide.

compared with rHuEpo intervention group, the expression of p-Akt and Bcl-2 in the LY294002 control group decreased, and the expression of Bad increased, there was no statistical difference $(\mathrm{P}>0.05)$. The intervention of rHuEpo, LY294002 and DMSO had no significant effect on the expression of Akt, and there was no statistical difference in the level of expression among groups $(\mathrm{P}>0.05)$.

\section{Discussion}

The persistent state of epilepsy is an emergency of the neurology department. If it is not treated in time, secondary to brain edema, brain hernia, respiratory and circulation system failure can cause persistent brain damage and cognitive impairment, and the mortality rate is $\sim 10-12 \%$ (8). In this study, it was used to improve the concentration of extracellular $\mathrm{K}^{+}$, depolarize the cell membrane and improve the excitatory PTZ as epileptogenic agent. There was no other neurotoxicity in PTZ itself. So it is a scientific choice to use PTZ as an epileptogenic agent to study neuron apoptosis damage attributed to SE. EEG and Racine grading were used to test the success of the SE model. Previous study shows that the damage of neurons after SE seizures is a mixed state of apoptosis, necrosis and coexistence of both, in which apoptosis is a very important damage form (9), but the mechanism of its occurrence is not clear. Some studies have observed that apoptotic neurons and apoptotic bodies appear in different degrees in the hippocampus of epileptic rats, while the activity of apoptosis-related factors enhanced, and the apoptotic cells with positive TUNEL staining appear in large numbers. In this study, the changes of apoptotic cells were observed by TUNEL staining in the PTZ kindled SE model rats. The results showed that a mass of epileptiform discharges were found in the EEG of the model group, and portion of the hippocampal neurons were necrosis and apoptosis, and the number of apoptotic cells increased more than that in the normal control group.

Mitochondria-dependent pathways play an important role in three of the main apoptotic-dependent pathways including mitochondria, death receptors and endoplasmic reticulum. The main sites and effective sites of the Bcl-2 family proteins are all on the mitochondrial membrane. The Bcl-2 family is the key to the integration of the signal to the cell mitochondria, which is called 'the doorman of apoptosis'. The members of Bcl-2 can form dipolymers, including homologous dipolymers or hetero-dipolymers, and these dipolymers play a vital role in the dynamic balance of cell survival and death, and then determine the fate of cells. The role of pro-apoptotic protein $\mathrm{Bad}$ is closely related to $\mathrm{Bcl}-2$ and Bcl-xL. Through the concentration-dependent form, Bad replaces the apoptotic protein $\mathrm{Bax}$ in the $\mathrm{Bcl}-2 / \mathrm{Bax}, \mathrm{Bcl}-\mathrm{xL} / \mathrm{Bax}$ dipolymer, which promotes the formation of a large amount of homologous dipolymers. When the homologous dipolymers content $>80 \%$, induced by apoptotic stimulation signal, programmed-death/ apoptosis appears in the cell. The ratio of Bax homologous dipolymers and hetero-dipolymers determine the survival of neurons, and Bad takes effect on promoting apoptosis by regulating this ratio. When the content of $\mathrm{Bcl}-\mathrm{xL} / \mathrm{Bax}$ and $\mathrm{Bcl}-2 / \mathrm{Bax}$ dipolymers in the cell is $\geq 50 \%$, the cells can resist apoptosis. Therefore, Bcl-2 and Bad are very important factors in Bcl-2 family. The changes of Bcl-2 and Bad were observed by immunohistochemistry, reverse transcription-PCR and western blotting in our study. The experimental results showed that the expressions of Bcl-2 and Bad immunoreactive cells, Bcl-2 mRNA, Bad mRNA and Bcl-2, Bad protein in the model group were significantly upregulated than the normal control group, which indicating PTZ activated Bad and Bcl-2 protein and mRNA increased significantly in the hippocampus neurons. The study of Henshall et al (10) and Meller et al (11) also found that Bcl-2 and Bad proteins may be activated and involved in neuronal apoptosis, which is consistent with our experimental results.

The in-depth study of pathophysiology of SE and the development of new effective therapeutic drugs are still difficult but are hot topics in world medical community now and even in the future. Cortex, hippocampus, astrocytes and brain capillary endothelial cells in brain tissue contain Epo receptors and can also produce endogenous Epo (12). Endogenous Epo needs full synthesis of protein and RNA transcription in neurons. Endogenous Epo production is inadequate in acute brain injury. The supply of exogenous Epo can reduce neuron damage. In this experiment, $\mathrm{rHuEpo}$ was used as a neuroprotective agent for intraperitoneal injection in the half hour after SE. The results showed that the intervention of rHuEpo could significantly reduce the occurrence of epileptic discharge, upregulate the expression of $\mathrm{Bcl}-2$ protein and mRNA in hippocampal neurons, downregulate the expression of apoptotic protein $\mathrm{Bad}$ and mRNA, suggesting that rHuEpo can regulate the expression level of these mitochondria apoptotic pathway related factors then reduce the necrosis and apoptosis of hippocampal 
neurons and play a neuroprotective role. Based on the above research, how rHuEpo regulates Bcl-2 and $\mathrm{Bad}$, and then achieves neuroprotective effect against apoptosis becomes our research direction.

The PI3K/Akt signal transduction pathway is a classic signal transduction pathway that has biological effect. It plays a multiple role in regulating cell growth, proliferation, differentiation and survival. PI3K/Akt signaling pathway is widespread in the nervous system, which can promote the survival of neurons through regulation and management of apoptosis and autophagy. Li et al (13) found that the improvement of ginseng protein in Alzheimer's disease was also mediated by activation of PI3K/Akt signaling pathway. Uzüm et al (14) found that Epo given through the abdominal cavity $24 \mathrm{~h}$ before the seizure would reduce the intensity of tonic clonic seizure and prolong the latent period of epileptic seizure. The activation of Akt induced by Epo is the key to exert its antiapoptotic effect. Akt is a direct downstream substrate of PI3K. The expression of p-Akt can infer the activation of PI3K/Akt pathway. In our study, we observed the changes of p-Akt by immunohistochemistry and western blotting to study the activation of PI3K/Akt pathway in SE. The results showed that rHuEpo increased the level of p-Akt in hippocampal neurons significantly, which suggests the neuroprotective effect of rHuEpo was closely related to the activation of PI3K/Akt pathway.

The role of PI3K/Akt pathway in promoting cell survival also have a series of important biological effects by regulating its downstream apoptosis-related proteins. Among them, the regulation of antiapoptotic protein $\mathrm{Bcl}-2$ and proapoptotic protein Bad in Bcl-2 family plays a significant role in nervous system diseases. After ischemic/anoxic stimulation, PI3K can be activated to phosphorylate Akt. P-Akt phosphorylates Bad in Ser136 and combines with chaperone 14-3-3 to form a complex. Bad is dissociated from heterogeneous dipolymer, which indirectly increases the expression of Bcl-2 and weakens the damage of apoptosis. Fu et al (15) found that $\mathrm{PI} 3 \mathrm{~K} / \mathrm{Akt} / \mathrm{Bad}$ signaling pathway played a role in apoptosis of $\mathrm{PC} 12$ cells induced by taxol. The expression level of Bcl-2 is regulated by $\mathrm{p}$-Akt, thus exerting neuroprotective effect (16). The results of knockout Akt mice showed that the level of $\mathrm{p}$-Akt decreased, the expression of $\mathrm{Bcl}-2$ protein in cytoplasm was reduced, and the damage of neurons was aggravated. The results of Miao et al (17) showed that the level of Bcl-2 expression was upregulated and the occurrence of apoptosis decreased, which might be achieved by enhancing PI3K/AKT signal transduction. Kong et al (18) found that the use of PI3K inhibitor LY294002 could prevent the protective effect due to increased p-Akt level. Xiao et al (19) studies showed that the expression of interleukin $1 \beta$ in the induced neurons could upregulate the expression of $\mathrm{p}$-Akt, and the length of the neuron dendrites could rapidly grow, and the expression of p-Akt was downregulated after the pretreatment of LY294002. In this experiment, we also carried out a more in-depth study to further observe whether rHuEpo regulates the mitochondrial apoptosis pathway related regulatory factors Bcl-2 and Bad via the PI3K/Akt pathway in the PTZ induced $\mathrm{SE}$ rat model, and offers neuroprotection. We used the PI3K inhibitor LY294002 to block it. The results showed that the protective effect of rHuEpo was obviously weakened when LY294002 was conducted before rHuEpo intervention. There was an increase in the occurrence of epileptic discharge in the LY294002 group and the apoptosis of the hippocampal neurons compared with that in the rHuEpo group, and the results of the immunodeficiency histochemistry and western blotting showed a decreased number of p-Akt- and Bcl-2-positive cells, and the expression of Bcl-2 mRNA and protein were also lower. The number of Bad-positive cells and the expression of Bad mRNA and protein were upregulated. It is further suggested that rHuEpo activates the important survival passageway PI3K/Akt pathway, which is related to improving the activity of Akt, and regulates the regulatory factors of Bcl-2 and Bad of mitochondrial apoptosis pathway through the PI3K/Akt signal transduction pathway to exert neuroprotective effect on antiapoptosis.

There are still some limitations in this study: the observation of large sample quantities without multi time-points. There are cross series between the signal paths and the complexities, and the PI3K/Akt path is not the only signal path of the Epo. How it works with the other channels to carry out 'cross talk' of neuroprotection is not clear. Thus more in-depth studies are required.

In conclusion, Epo directly or indirectly regulates the regulatory factors of $\mathrm{Bcl}-2$ and $\mathrm{Bad}$ of mitochondrial apoptosis pathway through PI3K/Akt signaling pathway, participates in the different stages of apoptosis, and then exerts neuroprotective effect on antiapoptosis and pro-survival. As a neuroprotective agent, Epo is widely used in the basic research of all kinds of nervous system diseases, such as closed brain injury, neonatal hypoxic encephalopathy and Alzheimer's disease. But the safe dose and treatment time of neuroprotection of Epo are different. The safety, dose dependence and time selectivity of Epo have attracted much attention. The study of Epo and formylated Epo is also being carried out to provide an important laboratory basis for the search for effective and feasible neuroprotective agents for the treatment of epilepsy.

\section{Acknowledgements}

Not applicable.

\section{Funding}

No funding was received.

\section{Availability of data and materials}

The datasets used and/or analyzed during the present study are available from the corresponding author on reasonable request.

\section{Authors' contributions}

JY and ZS were responsible for specimen collection and preparation. XS, YZ, BL and SW contributed to TUNEL and immunohistochemical staining. LJ, BZ, MZ and XF performed PCR. KY and WW helped with western blotting. All authors read and approved the final study.

\section{Ethics approval and consent to participate}

The study was approved by the Ethics Committee of The Second Hospital of Hebei Medical University (Hebei, China). 


\section{Patient consent for publication}

Not applicable.

\section{Competing interests}

The authors declare that they have no competing interests.

\section{References}

1. Wang C, Xie N, Wang Y, Li Y, Ge X and Wang M: Role of the mitochondrial calcium uniporter in rat hippocampal neuronal death after pilocarpine-induced status epilepticus. Neurochem Res 40: 1739-1746, 2015.

2. Buckmaster PS, Wen X, Toyoda I, Gulland FM and Van Bonn W: Hippocampal neuropathology of domoic acid-induced epilepsy in California sea lions (Zalophus californianus). J Comp Neurol 522: 1691-1706, 2014.

3. Wassink G, Davidson JO, Dhillon SK, Fraser M, Galinsky R, Bennet L and Gunn AJ: Partial white and grey matter protection with prolonged infusion of recombinant human erythropoietin after asphyxia in preterm fetal sheep. J Cereb Blood Flow Metab 37: 1080-1094, 2017

4. Wang M, Yan W, Liu Y, Hu H, Sun Q, Chen X, Zang W and Chen L: Erythropoietin ameliorates diabetes-associated cognitive dysfunction in vitro and in vivo. Sci Rep 7: 2801, 2017.

5. Vinberg M, Miskowiak K, Hoejman P,Pedersen Mand Kessing LV: The effect of recombinant erythropoietin on plasma brain derived neurotrophic factor levels in patients with affective disorders: A randomised controlled study. PLoS One 10: e0127629, 2015.

6. Bahçekapılı N, Akgün-Dar K, Albeniz I, Kapucu A, Kandil A, Yağıı O and Üzüm G: Erythropoietin pretreatment suppresses seizures and prevents the increase in inflammatory mediators during pentylenetetrazole-induced generalized seizures. Int J Neurosci 124: 762-770, 2014.

7. Ugurluer G, Cebi A, Mert H, Mert N, Serin M and Erkal HS: Neuroprotective effects of erythropoietin against oxidant injury following brain irradiation: An experimental study. Arch Med Sci 12: 1348-1353, 2016.

8. Liu J, Si Z, Li S, Huang Z, He Y, Zhang T and Wang A: The calcineurin inhibitor FK506 prevents cognitive impairment by inhibiting reactive astrogliosis in pilocarpine-induced status epilepticus rats. Front Cell Neurosci 11: 428, 2018.

9. Mikati MA, Abi-Habib RJ,El Sabban ME, Dbaibo GS, Kurdi RM, Kobeissi M, Farhat F and Asaad W: Hippocampal programmed cell death after status epilepticus: Evidence for NMDA-receptor and ceramide-mediated mechanisms. Epilepsia 44: 282-291, 2003
10. Henshall DC, Araki T, Schindler CK, Lan J-Q, Tiekoter KL, Taki W and Simon RP: Activation of Bcl-2-associated death protein and counter-response of Akt within cell populations during seizure-induced neuronal death. J Neurosci 22: 8458-8465, 2002.

11. Meller R, Schindler CK, Chu XP, Xiong ZG, Cameron JA, Simon RP and Henshall DC: Seizure-like activity leads to the release of BAD from 14-3-3 protein and cell death in hippocampal neurons in vitro. Cell Death Differ 10: 539-547, 2003

12. Genc S, Koroglu TF and Genc K: Erythropoietin as a novel neuroprotectant. Restor Neurol Neurosci 22: 105-119, 2004.

13. Li H, Kang T, Qi B, Kong L, Jiao Y, Cao Y, Zhang J and Yang J: Neuroprotective effects of ginseng protein on PI3K/Akt signaling pathway in the hippocampus of $\mathrm{D}$-galactose/ $\mathrm{AlCl} 3$ inducing rats model of Alzheimer's disease. J Ethnopharmacol 179: 162-169, 2016.

14. Uzüm G, Sarper Diler A, Bahçekapili N and Ziya Ziylan Y: Erythropoietin prevents the increase in blood-brain barrier permeability during pentylentetrazol induced seizures. Life Sci 78: 2571-2576, 2006.

15. Fu Z, Yang J, Wei Y and Li J: Effects of piceatannol and pterostilbene against $\beta$-amyloid-induced apoptosis on the $\mathrm{PI} 3 \mathrm{~K} / \mathrm{Akt} / \mathrm{Bad}$ signaling pathway in PC12 cells. Food Funct 7: 1014-1023, 2016

16. Wang C, Wang Z, Zhang X, Zhang X, Dong L, Xing Y, Li Y, Liu Z, Chen L, Qiao H, et al: Protection by silibinin against experimental ischemic stroke: Up-regulated pAkt, pmTOR, HIF- $1 \alpha$ and Bcl-2, down-regulated Bax, NF- $\kappa \mathrm{B}$ expression. Neurosci Lett 529: 45-50, 2012.

17. Miao J, Wang L, Zhang X, Zhu C, Cui L, Ji H, Liu Y and Wang X: Protective effect of aliskiren in experimental ischemic stroke: Up-regulated p-PI3K, p-AKT, Bcl-2 expression, Attenuated Bax Expression. Neurochem Res 41: 2300-2310, 2016.

18. Kong J, Ren G, Jia N, Wang Y, Zhang H, Zhang W, Chen B and Cao Y: Effects of nicorandil in neuroprotective activation of PI3K/AKT pathways in a cellular model of Alzheimer's disease. Eur Neurol 70: 233-241, 2013.

19. Xiao Z, Peng J, Yang L, Kong H and Yin F: Interleukin-1ß plays a role in the pathogenesis of mesial temporal lobe epilepsy through the PI3K/Akt/mTOR signaling pathway in hippocampal neurons. J Neuroimmunol 282: 110-117, 2015.

This work is licensed under a Creative Commons Attribution-NonCommercial-NoDerivatives 4.0 International (CC BY-NC-ND 4.0) License. 